\title{
THE BREADTH AND DIMENSION OF A TOPOLOGICAL LATTICE
}

TAE HO CHOE

E. Dyer and A. Shields [7] conjectured that if $L$ is a compact connected metrizable distributive topological lattice, then $\operatorname{dim}(L)$ is equal to the breadth of $L$. L. W. Anderson [1] has proved that if $L$ is a locally compact, (chain-wise) connected distributive topological lattice then the breadth of $L$ is less then or equal to the codimension of $L$.

In this note we shall show that if $L$ is a locally compact connected distributive topological lattice of inductive dimension $n$ and if the set of points at which $L$ has dimension $n$ has nonvoid interior, then the breadth of $L$ is also $n$.

Our terminology and notation used in this paper are the same as in $[1]$ and $[4]$.

It is well known that the number of elements in a finite Boolean algebra is always a power of two, and if there are $2^{n}$ elements then there are exactly $n$ atoms.

It is also known that the center $C$ of any lattice with 0 and 1 forms a Boolean lattice with the same 0 and 1 , and that if $L$ is a compact connected topological lattice of codimension $n$ then the cardinal of $C$ of $L$, hereafter denoted by $\operatorname{Card}(\operatorname{Cen}(L))$, is at most $2^{n}$, [4].

The following two lemmas have appeared in [4].

Lemma 1. Let $L$ be a compact connected distributive topological lattice. Then $L$ is iseomorphic (homeomorphic and lattice-isomorphic) with a Cartesian product of $n$ nondegenerate compact connected chains (hereafter called an $n$-cube) iff (i) the center of $L$ has exactly $n$ atoms and (ii) for each atom $x$ of the center $x \wedge L$ is a chain.

Lemma 2. Let $L$ be a distributive topological lattice with 0 and 1 . If $\left\{x_{1}, \cdots, x_{n}\right\}$ is the set of all the atoms of the center of $L$ and if $x$ and $y$ are two incomparable elements in $x_{i} \wedge L$ for some $i$, then $\operatorname{Card}(\operatorname{Cen}(L))$ $<\operatorname{Card}\left(\operatorname{Cen}\left(\left[x \wedge y, x \vee y \vee c\left(x_{i}\right)\right]\right)\right)$, where $c\left(x_{i}\right)$ is the complemented element of $x_{i}$ in the center of $L$.

Hereafter, inductive dimension is referred to as dimension. In a space $L$ of dimension $n, L_{n}$ denotes the set of all points at which $L$ has dimension $n$. If $L$ is a topological lattice and $[x, y] \subset L$, and if $\operatorname{Card}(\operatorname{Cen}([x, y]))=2^{n}$, then $\mathrm{Ca}([x, y])$ will denote the cardinal $n$ of the atoms of $\operatorname{Cen}([x, y])$. It is known [6] that the codimension is

Received by the editors June 17, 1968. 
less than or equal to the inductive dimension in a locally compact Hausdorff space.

THEOREM. If $L$ is a locally compact connected distributive topological lattice of dimension $n$ and if $L_{n}$ has a nonvoid interior, then $L$ contains a subset which is iseomorphic with an n-cube.

Proof. Let $W_{1}$ be a nonvoid open subset of $L$ and $W_{1} \subset L_{n}$. For an element $w \in W_{1}$ choose neighborhoods $V_{1}$ and $U_{1}$ of $w$ and a closed interval $[a, b]$ such that $V_{1}$ is convex, $U_{1}^{*}$ compact and $V_{1} \subset[a, b]$ $\subset U_{1}^{*} \subset W_{1}$, (see [5]), where $U_{1}^{*}$ denotes the closure of $U_{1}$. Then $M=[a, b]$ is a compact connected distributive topological lattice of dimension $n$ in its relative topology. Consider $N=\{m \mid m=\mathrm{Ca}([x, y])$ for a closed interval $\left.[x, y] \subset V_{1}\right\}$. Then $N$ is bounded by $n$. Let $m$ be the greatest integer of $N, m=\mathrm{Ca}([\alpha, \beta])$, and let $\left\{x_{1}, \cdots, x_{m}\right\}$ be the set of atoms of the center of $[\alpha, \beta]$. Now we shall show that for each $i, I_{i}=x_{i} \wedge[\alpha, \beta]$ is a nondegenerate compact connected chain. In fact, $[\alpha, \beta]$ is a distributive sublattice. Suppose that $I_{i}$ is not a chain for some $i$. Then, by Lemma 2 , there exists a closed interval $\left[\alpha^{\prime}, \beta^{\prime}\right]$ in $[\alpha, \beta]$ such that $m<\mathrm{Ca}\left(\left[\alpha^{\prime}, \beta^{\prime}\right]\right)$. This is a contradiction. For each $i, I_{i}$ is compact and connected because $[\alpha, \beta]$ is. Moreover, the relative topology of each $I_{i}$ is its intrinsic topology, (see [3]). Since $M$ is compact, $[\alpha, \beta]$ is a compact connected distributive topological lattice in its relative topology. By Lemma 1 , it follows that $[\alpha, \beta]$ is iseomorphic with an $m$-cube $I^{m}=I_{1} \times I_{2} \times \cdots \times I_{m}$ under a mapping $f$.

Suppose $m<n$. Let $p$ be an element of $[\alpha, \beta]$ such that the $i$ th coordinate of $f(p)$ is an inner point of $I_{i}, i=1,2, \cdots, m$, (such a point $p$ is called an inner point of $\left.I^{m}\right)$. Now we choose a convex neighborhood $U_{2}$ of $p$ such that

$$
U_{2} \subset L \backslash \cup\{(c \wedge L) \cup(c \vee L) \mid c \in \operatorname{Cen}([\alpha, \beta]) \text { and } c \neq \alpha, c \neq \beta\} .
$$

Setting $U=U_{2} \cap V_{1}$, we choose a neighborhood $W$ of $p$ such that $W \vee W \subset U$ and $W \wedge W \subset U$. If we set $V=U \cap[\alpha, \beta]$, then $V$ is a convex neighborhood of $p$ in $[\alpha, \beta]$, and the dimension of $V$ is at most $m$. Thus $W \backslash V \neq \square$. For an element $z \in W \backslash V$ we have either $z \bigvee p \notin V$ or $z \wedge p \notin V$ because, otherwise, $z \in V$. Let us first assume that $z \bigvee p \notin V$. But $z \bigvee p \in U$. Set $\gamma=(z \bigvee p) \wedge \beta$. Then $p \leqq \gamma<z \vee p$. Since $U$ is convex and contains $p$ and $z \bigvee p$, we have $\gamma \in V=U \cap[\alpha, \beta]$. Clearly $\gamma$ is an inner point of $I^{m}$. So $[\gamma, \beta]$ is also iseomorphic with a sub $m$-cube $J^{m}$ of $I^{m}$. Consider a compact connected chain $C(\gamma, \delta)$ from $\gamma$ to $\delta=z \bigvee p$. Then $C(\gamma, \delta)$ is nondegenerate. Moreover, we have $\delta \wedge[\gamma, \beta]=\{\gamma\}$. Therefore, the mapping $g: Q^{m+1}=[\gamma, \beta] \times C(\gamma, \delta)$ 
$\rightarrow P^{m+1}=[\gamma, \beta] \vee C(\gamma, \delta)$ defined by $g(x, s)=x \vee s$ is an iseomorphism, where $g^{-1}(y)=(y \wedge \beta, y \wedge \delta)$. Since $g^{-1}\left(U \cap P^{m+1}\right)$ is nonvoid and open in $Q^{m+1}$, and $Q^{m+1}$ is iseomorphic with an $(m+1)$-cube $J^{m} \times C(\gamma, \delta)$, $U \cap P^{m+1}$ contains a closed interval which is iseomorphic with an $(m+1)$-cube, and which is contained in $V_{1}$. Moreover, $\mathrm{Ca}\left(J^{m} \times C(\gamma, \delta)\right)$ $=m+1$. This is a contradiction to the fact of the maximality of $m$. For the case that $z \wedge p \notin V$, the dual argument also leads to a contradiction. Hence we have $m=n$ which completes the proof.

COROLlary 1. Under the same hypothesis of the Theorem, the breadth, the dimension and the codimension are all the same.

It is known [8] that if $L$ is a compact separable space of dimension $n$, then $L_{n}$ has dimension $n$, and that any $n$-dimensional subset of Euclidean $n$-space has novoid interior.

Hence we have the following corollary:

COROLlary 2. If $L$ is a locally compact connected distributive topological lattice of dimension $n$ which is topologically contained in Euclidean $n$-space, then $L$ contains a subset which is iseomorphic with an $n$-cell.

REMARK. It is a natural question that if $L$ is a compact connected distributive topological lattice of dimension $n$, then $L_{n}$ has nonvoid interior. This question is still open. Professor J. T. Borrego has, however, given an example of a compact connected, locally connected, acyclic topological semilattice of 2-dimensions whose $L_{2}$ has void interior.

\section{REFERENCES}

1. L. W. Anderson, On the breadth and codimension of a topological lattice, Pacific J. Math. 9 (1959), 329-333.

2. - One dimension topological lattices, Proc. Amer. Math. Soc. 10 (1959), 715-720.

3. - On the distributivity and simple connectivity of plane topological lattices, Trans. Amer. Math. Soc. 91 (1959), 102-112.

4. T. H. Choe, On compact topological lattice of finite dimension, Trans. Amer. Math. Soc. 140 (1969), 223-237.

5. - Intrinsic topologies in a topological lattice, Pacific J. Math. 28 (1969).

6. H. Cohen, A cohomological definition of dimension for locally compact Hausdorff spaces, Duke Math. J. 21 (1954), 209-224.

7. E. Dyer and A. Shields, Connectivity of topological lattices, Pacific J. Math. 9 (1959), 443-448.

8. W. Hurewicz and H. Wallman, Dimension theory, Princeton Univ. Press, Princeton, N. J., 1948.

UNIVERSity OF MASSACHUSETTS AND MCMASTER UNIVERSITY 